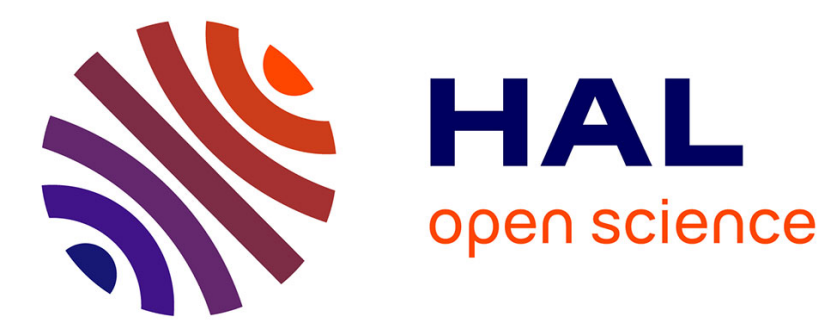

\title{
Influence de la biréfringence due aux défauts natifs sur l'effet électro-optique du ZnSe monocristallin
}

\author{
P. Bugnet, J. Baillou, J. Daunay, Jac. Daunay
}

\section{To cite this version:}

P. Bugnet, J. Baillou, J. Daunay, Jac. Daunay. Influence de la biréfringence due aux défauts natifs sur l'effet électro-optique du ZnSe monocristallin. Revue de Physique Appliquée, 1979, 14 (1), pp.259-263. 10.1051/rphysap:01979001401025900 . jpa-00244585

\section{HAL Id: jpa-00244585 https://hal.science/jpa-00244585}

Submitted on 1 Jan 1979

HAL is a multi-disciplinary open access archive for the deposit and dissemination of scientific research documents, whether they are published or not. The documents may come from teaching and research institutions in France or abroad, or from public or private research centers.
L'archive ouverte pluridisciplinaire HAL, est destinée au dépôt et à la diffusion de documents scientifiques de niveau recherche, publiés ou non, émanant des établissements d'enseignement et de recherche français ou étrangers, des laboratoires publics ou privés. 


\title{
Influence de la biréfringence due aux défauts natifs sur l'effet électro-optique du ZnSe monocristallin
}

\author{
P. Bugnet, J. Baillou, J. Daunay et Jac. Daunay \\ Laboratoire d'Optoélectronique, Faculté des Sciences \\ 40, avenue du Recteur-Pineau, 86022 Poitiers Cedex, France \\ (Reçu le 30 juin 1978, révisé le 10 octobre 1978, accepté le 10 octobre 1978)
}

\begin{abstract}
Résumé. - Les monocristaux de ZnSe préparés par condensation de la phase vapeur sont de structure cubique mais présentent des défauts de structure qui les rendent légèrement biréfringents. L'influence de cette biréfringence sur l'effet électro-optique linéaire est étudiée expérimentalement pour diverses orientations du champ électrique. La dispersion des lignes neutres avec le champ est mise en évidence et on recherche les conditions susceptibles de la minimiser.
\end{abstract}

\begin{abstract}
In spite of their cubic structure, vapour grown $\mathrm{ZnSe}$ single crystals exhibit a weak birefringence due to structural defects. Its influence on the linear electro-optic effect is studied for several directions of the electric field. Investigations on the dispersion of the active axes with the field are carried out, and an attempt is made to find the orientation which minimize it.
\end{abstract}

1. Introduction. - Le séléniure de zinc monocristallin, de structure cubique (blende), présente dans le visible un effet électro-optique linéaire relativement élevé qui permet d'envisager son emploi comme modulateur optique. Les caractéristiques générales de la biréfringence induite par un champ électrique dans les différentes classes cristallines sont bien connues et ont été maintes fois exposées [1-5]. Cependant certains cristaux présentent des défauts, ou des contraintes résiduelles, qui perturbent l'effet électro-optique [6-8]. Nous avons étudié le cas des monocristaux de $\mathrm{ZnSe}$ préparés par croissance à partir de la phase vapeur, qui présentent des défauts de structure d'une symétrie bien définie, et sont de ce fait légèrement biréfringents en l'absence de toute excitation électrique. Il en résulte notamment une dispersion des lignes neutres avec le champ électrique appliqué, susceptible de fausser la relation entre le déphasage des deux ondes transmises et le champ.

2. Influence de la rotation des lignes neutres sur la modulation d'un faisceau lumineux. - Lorsque le cristal est placé entre polariseur et analyseur croisés, le polariseur faisant un angle $\beta$ avec l'une des lignes neutres, l'intensité lumineuse transmise est :

$I_{\mathrm{T}}=I_{0} \sin ^{2} 2 \beta \sin ^{2} \frac{\Phi}{2}$

où $\Phi$ est le déphasage entre les deux ondes transmises par le cristal.
Si la modulation du champ électrique produit seulement une modulation de $\Phi$, sans modifier la position des lignes neutres, à une variation $\Delta E$ du champ correspond une variation de l'intensité transmise :

$$
\Delta I_{\mathrm{T}}=\left(\frac{I_{0}}{2} \sin ^{2} 2 \beta \sin \Phi\right) \Delta \Phi .
$$

En particulier lorsque $\Phi$ est proportionnel à $E$ :

$\Delta I_{\mathrm{T}}=\left(\frac{I_{0}}{2} \sin ^{2} 2 \beta \sin \Phi\right) k \Delta E$.

Par contre, si la position des lignes neutres dépend du champ :

$$
\begin{aligned}
& \Delta I_{\mathrm{T}}=\left(\frac{I_{0}}{2} \sin ^{2} 2 \beta \sin \Phi\right) \Delta \Phi+ \\
& +\left(2 I_{0} \sin 4 \beta \sin ^{2} \frac{\Phi}{2}\right) \Delta \beta .
\end{aligned}
$$

Il n'y a plus de relation simple entre la modulation du champ et celle de l'intensité lumineuse, d'où l'intérêt d'utiliser une configuration dans laquelle la position des lignes neutres est indépendante du champ.

3. Effet électro-optique d'un cristal de structure blende. - Un tel cristal est optiquement isotrope. Sous l'action d'un champ électrique $E$, il devient 
biréfringent, le tenseur d'imperméabilité prenant la forme générale suivante dans le système d'axes habituels du cubique [4] :

$B=\left[\begin{array}{lll}B_{0} & r_{41} E_{z} & r_{41} E_{y} \\ r_{41} E_{z} & B_{0} & r_{41} E_{x} \\ r_{41} E_{y} & r_{41} E_{x} & B_{0}\end{array}\right]$

$B_{0}=1 / n_{0}^{2}, n_{0}$ étant l'indice du $\mathrm{ZnSe}$ cubique, et $r_{41}$ est la valeur commune des 3 coefficients électrooptiques non nuls $\left(r_{41}=r_{52}=r_{63}\right)$.

Il en résulte que le cristal est généralement biaxe, mais devient uniaxe si le champ $E$ est parallèle à l'axe cristallographique [111] qui devient l'axe optique.

Habituellement, [5], le champ $E$ est parallèle à l'axe [110] (les plans (110) sont les plans de clivage naturel). Les axes de l'ellipsoïde des indices, ou directions propres du tenseur d'imperméabilité, sont alors respectivement (Fig. 1) :

- l'axe cristallographique [1̄ㅡㅇ,

- deux directions du plan (11̄0) situées à 45 degrés de part et d'autre de l'axe [001].

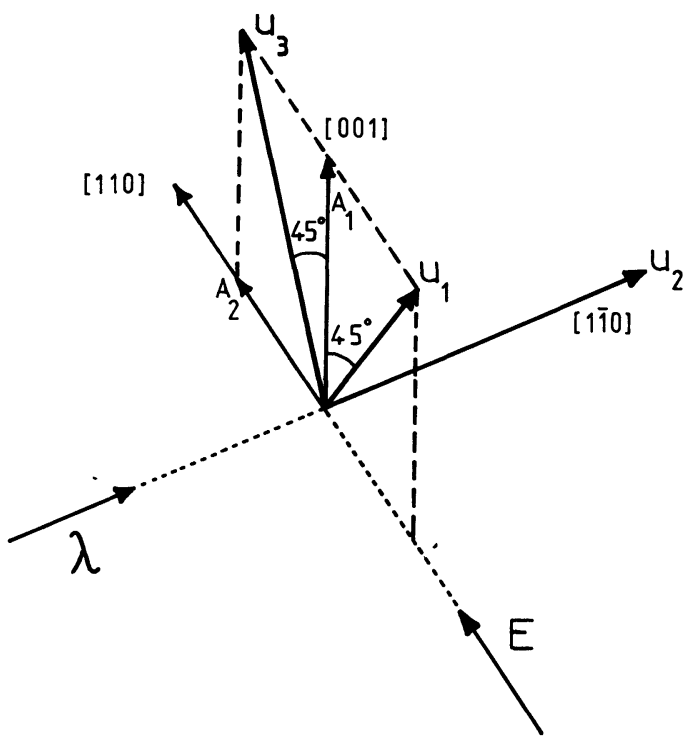

Fig. 1. - Axes $\left(u_{1}, u_{2}, u_{3}\right)$ de l'ellipsoïde des indices pour l'effet électro-optique linéaire de la blende. Champ électrique dans la direction [110]. Propagation de la lumière dans la direction [1 $\overline{10}$ ]. $A_{1}$ et $A_{2}$ sont les deux axes optiques.

[Index ellipsoid axes $\left(u_{1}, u_{2}, u_{3}\right)$ for the linear electro-optic effect in zinc blende structure when electric field is applied along the [110] direction. Light propagation along the [1ㅣㅣ direction. $A_{1}$ and $A_{2}$ axes are optic axes.]

Les deux axes optiques $A_{1}$ et $A_{2}$ sont respectivement les directions [001] et [110]. Les axes de l'ellipsoïde des indices sont indépendants de l'intensité du champ et de la longueur d'onde. Dans ces conditions,

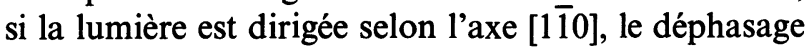
entre les deux ondes transmises est :

$\Phi=\frac{2 \pi l}{\lambda} n_{0}^{3} r_{41} E=k E$.
Nous avons également appliqué le champ $E$ parallèlement à l'axe [001]. Dans ce cas les axes de l'ellipsoïde des indices sont les axes cristallographiques [001], [110] et [1 $\overline{1} 0]$ (Fig. 2).

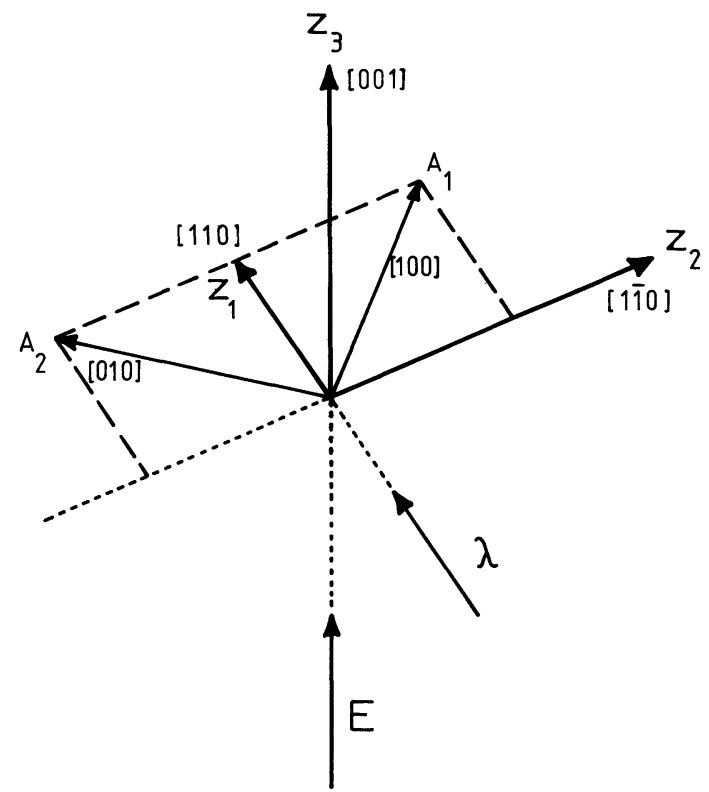

Fig. 2. - Axes $\left(z_{1}, z_{2}, z_{3}\right)$ de l'ellipsoïde des indices pour l'effet électro-optique linéaire de la blende. Champ électrique dans la direction [001]. Propagation de la lumière dans la direction [110]. Les axes optiques $A_{1}$ et $A_{2}$ sont situés sur les deux axes cristallographiques [100] et [010].

[Index ellipsoid axes $\left(z_{1}, z_{2}, z_{3}\right)$ for the linear electro-optic effect in zinc blende structure when electric field is applied along the [001] direction. Light propagation along the [110] direction. Optic axes $A_{1}$ and $A_{2}$ are located on the crystallographic axes [100] and [010].]

Les axes optiques sont parallèles aux directions [100] et [010]. Lorsque la lumière est dirigée selon les

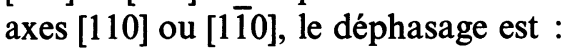

$\Phi=\frac{\pi l}{\lambda} n_{0}^{3} r_{41} E$

donc deux fois plus faible que dans le cas précédent.

4. Biréfringence due aux défauts dans ZnSe préparé en phase vapeur. - Les cristaux préparés par sublimation de poudre de $\mathrm{ZnSe}$ et condensation cristallisent dans le système de la blende [9]. Cependant, ils présentent des défauts de structure qui leur confèrent une biréfringence faible, mais du même ordre de grandeur que la biréfringence due à un champ électrique $[5,10]$. L'observation au microscope polarisant de cette biréfringence accidentelle montre qu'elle n'est pas répartie uniformément [11]. Pour les études relatées ici, les cristaux ont été sélectionnés dans des zones présentant un aspect homogène au microscope polarisant, et montés dans des supports avec diaphragme pour exclure toute autre biréfringence due 
à des contraintes locales, en particulier près des arêtes. Avec ces précautions, des résultats pratiquement identiques ont été obtenus pour les 6 échantillons étudiés.

L'étude de la biréfringence accidentelle [11] selon plusieurs directions cristallographiques a permis de préciser :

- la position des lignes neutres sur les 3 faces (110), (110) et (001) des cristaux,

- l'amplitude $\Delta n$ et la dispersion de la biréfringence selon les 3 directions de propagation [110], [1iㅣ] et [001].

On constate une dispersion des lignes neutres avec la longueur d'onde lorsque la lumière est dirigée suivant l'axe [110], mais non lorsqu'elle est dirigée suivant une direction perpendiculaire, [001] ou [110]. Cette dispersion montre que les cristaux sont naturellement biaxes. On note également une rotation des axes optiques quand la longueur d'onde varie, pour une propagation de la lumière dans la direction [110]. Les axes de l'ellipsoïde des indices sont respectivement (Fig. 3) :

- l'axe [110], noté $o x_{2}^{\prime}$,

- deux directions du plan (110) qui varient avec la longueur d'onde, notées $o x_{1}^{\prime}$ et $o x_{3}^{\prime}$.

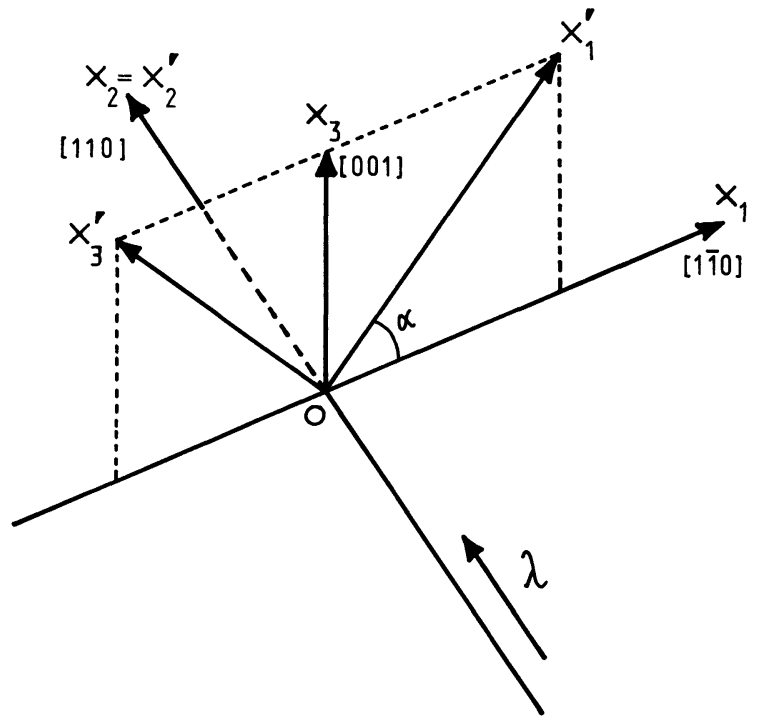

Fig. 3. - Axes $\left(x_{1}^{\prime}, x_{2}^{\prime}, x_{3}^{\prime}\right)$ de l'ellipsoïde des indices pour la biréfringence due aux défauts natifs dans $\mathrm{ZnSe}$.

[Index ellipsoid axes $\left(x_{1}^{\prime}, x_{2}^{\prime}, x_{3}^{\prime}\right)$ for the birefringence due to the native defects in $\mathrm{ZnSe}$.]
Il existe une longueur d'onde particulière $\lambda_{0}$ (4 $930 \AA \pm 10 \AA$ suivant les échantillons) pour laquelle la biréfringence est nulle à la fois dans les deux directions [1 $1 \overline{1} 0]$ et [001] mais pas dans la direction [110]. Dans ce cas les axes optiques se confondent avec les axes [1ํㅣ] et [001], arêtes des échantillons.

5. Effet électro-optique d'un cristal de ZnSe, champ électrique dans la direction [110]. - La présence des défauts peut être exprimée en ajoutant au terme général du tenseur d'imperméabilité $B_{i j}=B_{0 i j} \delta_{i j}$ du $\mathrm{ZnSe}$ cubique un terme correctif $d_{i j}$. En présence d'un champ électrique ce terme général devient au premier ordre :

$B_{i j}+\Delta B_{i j}$

avec

$\Delta B_{i j}=d_{i j}+\frac{\partial B_{i j}}{\partial E_{k}} E_{k}+\frac{\partial d_{i j}}{\partial E_{k}} E_{k}+\cdots$

ou encore :

$\Delta B_{i j}=d_{i j}+e_{i j}+c_{i j}$

$d_{i j}$ est la perturbation du tenseur de la blende par les défauts, $e_{i j}$ la perturbation de la blende par le champ électrique, $c_{i j}$ un terme de couplage champdéfauts. $d_{i j}$ et $e_{i j}$ sont très petits $(\Delta n$ de l'ordre de $10^{-5}$ ) devant $B_{0}$, et $c_{i j}$ est un terme du $2^{\mathrm{e}}$ ordre que nous négligerons.

Par suite de l'orientation de l'ellipsoïde des indices de la biréfringence due aux défauts, indiquée figure 3, le tenseur $d_{i j}$ a la forme suivante sur la base $\left(x_{1} x_{2} x_{3}\right)$ :

$\left[d_{i j}\right]_{\left(x_{1} x_{2} x_{3}\right)}=\left[\begin{array}{lll}d_{1} & 0 & d_{3} \\ 0 & d_{2} & 0 \\ d_{3} & 0 & d_{1}\end{array}\right]$

et par changement d'axes, sur la base $\left(u_{1} u_{2} u_{3}\right)$ des directions propres de $e_{i j}$ (Fig. 1) :

$\left[d_{i j}\right]_{\left(u_{1} u_{2} u_{3}\right)}=\left[\begin{array}{ccc}\frac{d_{1}+d_{2}}{2} & \frac{d_{3}}{\sqrt{2}} & \frac{d_{1}-d_{2}}{2} \\ \frac{d_{3}}{\sqrt{2}} & d_{1} & \frac{d_{3}}{\sqrt{2}} \\ \frac{d_{1}-d_{2}}{2} & \frac{d_{3}}{\sqrt{2}} & \frac{d_{1}+d_{2}}{2}\end{array}\right]$

Finalement, le tenseur d'imperméabilité devient, au $1^{\mathrm{er}}$ ordre, sur cette base :

$\left[B_{i j}\right]_{\left(u_{1} u_{2} u_{3}\right)}=\left[\begin{array}{ccc}B_{0}+\frac{d_{1}+d_{2}}{2}+e & \frac{d_{3}}{\sqrt{2}} & \frac{d_{1}-d_{2}}{2} \\ \frac{d_{3}}{\sqrt{2}} & B_{0}+d_{1} & \frac{d_{3}}{\sqrt{2}} \\ \frac{d_{1}-d_{2}}{2} & \frac{d_{3}}{\sqrt{2}} & B_{0}+\frac{d_{1}+d_{2}}{2}-e\end{array}\right]$ avec $B_{0}=1 / n_{0}^{2}$ et $e=r_{41} E$. 
Les directions propres de ce tenseur sont à priori quelconques, fonctions à la fois du champ $E$ et de la longueur d'onde $\lambda$ (par l'intermédiaire des $d_{i}$ ). L'observation expérimentale avec la géométrie habituelle, lumière dirigée selon l'axe [1iㅣㄹ, fait apparaître une forte dispersion des lignes neutres avec le champ (Fig. 4). Pour les valeurs très élevées du champ, les lignes neutres tendent vers la position théorique correspondant à l'absence de défauts. Par ailleurs la dispersion avec le champ est minimale pour la longueur d'onde $\lambda_{0}=4930 \AA$ pour laquelle les deux indices de la biréfringence due aux défauts sont égaux dans la direction [110].

6. Effet électro-optique d'un cristal de $\mathrm{ZnSe}$, champ électrique dans la direction [001]. - En utilisant la base $\left(x_{1} x_{2} x_{3}\right)$ nous obtenons cette fois :

$\left[e_{i j}\right]_{\left(x_{1} x_{2} x_{3}\right)}=\left[\begin{array}{rrr}-e & 0 & 0 \\ 0 & e & 0 \\ 0 & 0 & 0\end{array}\right]$

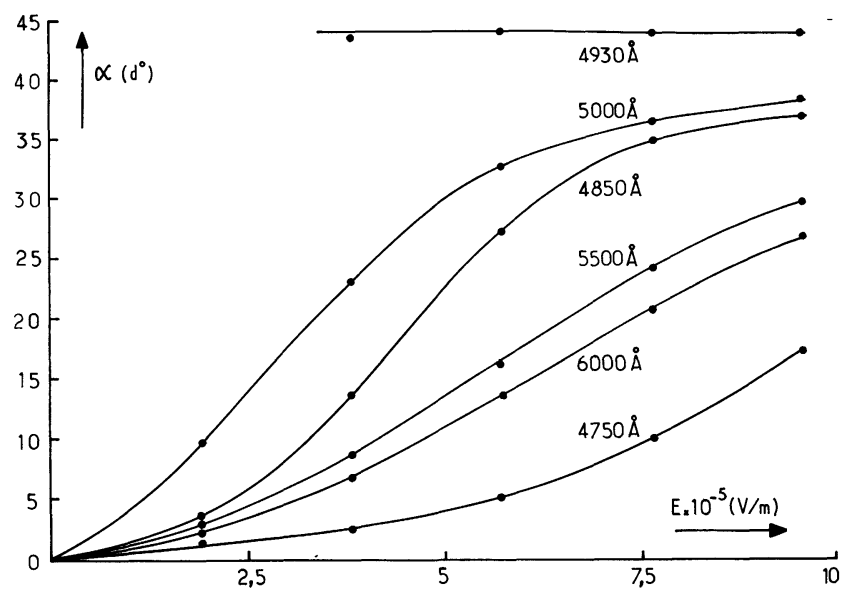

Fig. 4. - Rotation des lignes neutres avec le champ électrique à différentes longueurs d'onde. Champ dans la direction [110]. Propagation de la lumière dans la direction [1ํㅣ.

[Active axes rotation versus electric field amplitude for several wavelengths, when the field is applied along the [110] direction. Light propagation along the [1ㅣㅣ direction.]'

avec $e=r_{41} E$ et

$\left[B_{i j}\right]_{\left(x_{1} x_{2} x_{3}\right)}=\left[\begin{array}{ccc}B_{0}+d_{1}-e & 0 & d_{3} \\ 0 & B_{0}+d_{2}+e & 0 \\ d_{3} & 0 & B_{0}+d_{1}\end{array}\right]$.

Ce tenseur admet l'axe [110] comme direction propre, les deux autres directions propres étant situées dans le plan (110). Par conséquent, en éclairant le cristal dans une direction perpendiculaire à l'axe [110], par exemple [1 $1 \overline{1} 0]$, on n'observera qu'une dispersion très faible des lignes neutres, due uniquement aux termes du $2^{\mathrm{e}}$ ordre que nous avons négligés. C'est ce que montrent les courbes expérimentales de la figure 5 .

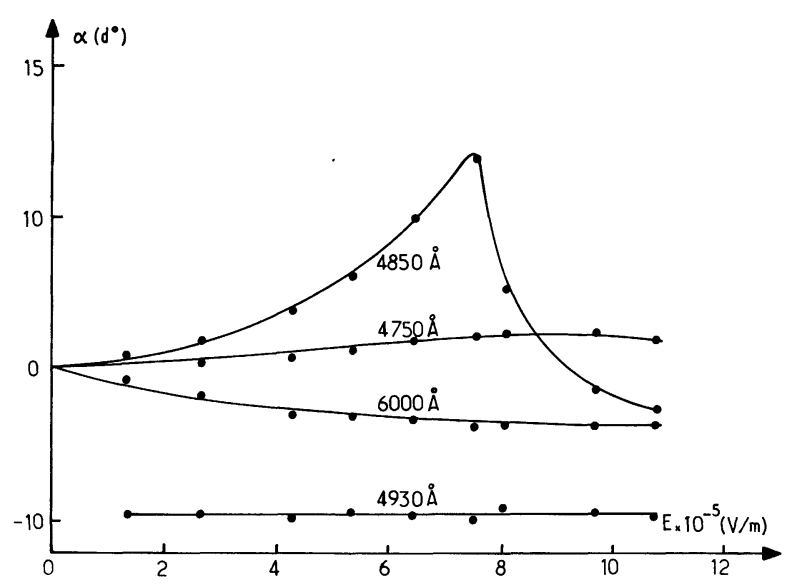

Fig. 5. - Rotation des lignes neutres avec le champ électrique pour différentes longueurs d'onde. Champ dans la direction [001].

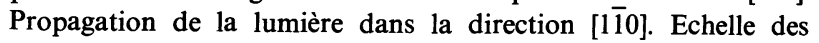
ordonnées dilatée par rapport à la figure 4 .

[Active axes rotation versus electric field amplitude for several wavelengths, when the field is applied along the [001] direction. Light propagation along the [1ㅣㅣ $]$ direction. The scale of angle axis is unlarged with respect to the figure 4 . ]
En particulier pour la longueur d'onde $\lambda_{0}$, les lignes neutres ne varient pratiquement pas de plus de 1 degré entre 0 et $10^{6} \mathrm{~V}$ par mètre.

Le déphasage entre les deux ondes transmises est alors sensiblement :

$\Phi=\frac{\pi l}{\lambda} n_{0}^{3} r_{41} E$

La dispersion des lignes neutres avec la longueur d'onde est également très faible, sauf pour les longueurs d'onde voisines du seuil d'absorption bande à bande (Fig. 6).

Un résultat similaire est obtenu lorsque le champ

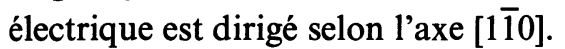

7. Influence des termes du deuxième ordre. - Il est possible d'obtenir une meilleure approche du tenseur d'imperméabilité en tenant compte des termes $\mathrm{du} 2^{\mathrm{e}}$ ordre $c_{i j}$ que nous avons négligés. La biréfringence due aux défauts présente les caractéristiques de la biréfringence d'un cristal monoclinique, le plan de symétrie étant le plan (110) de la blende. En utilisant le tenseur électro-optique du système monoclinique, on constate que lorsque $E$ est dirigé selon l'axe [001], $\left[c_{i j}\right]$ est de la forme :

$\left[c_{i j}\right]_{\left(x_{1} x_{2} x_{3}\right)}=\left[\begin{array}{lll}c_{11} & 0 & c_{13} \\ 0 & c_{22} & 0 \\ c_{13} & 0 & c_{33}\end{array}\right]$ 


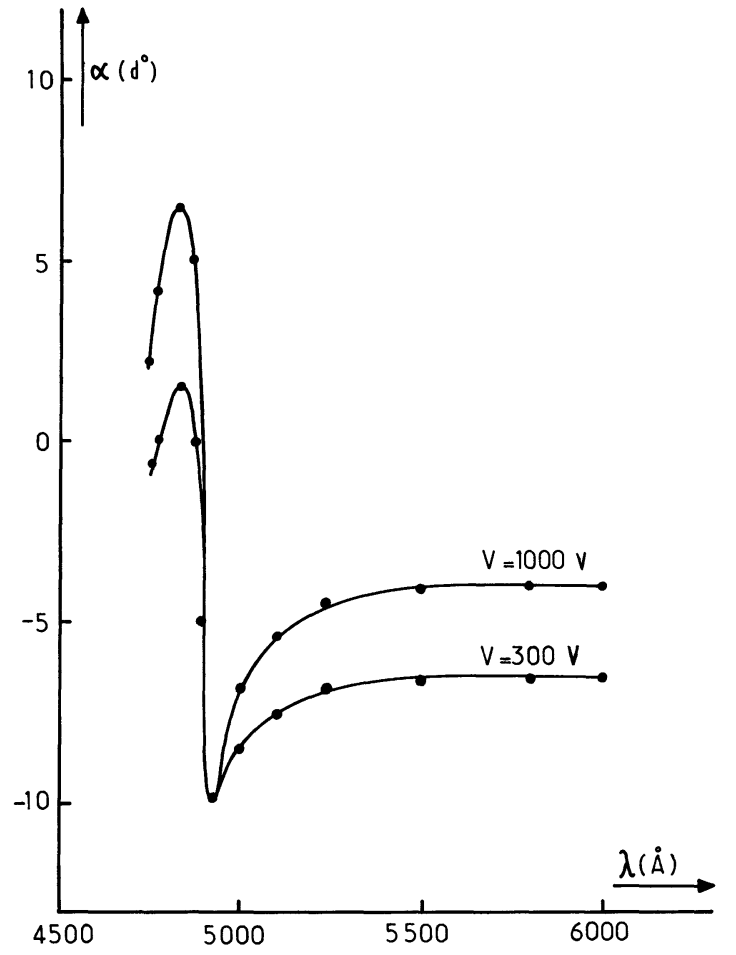

Fig. 6. - Rotation des lignes neutres avec la longueur d'onde pour différentes valeurs du champ électrique dirigé selon l'axe [001]. Propagation de la lumière dans la direction [110].

[Active axes rotation versus wavelength for several electric fields applied along the [001] axis. Light propagation along the [110] direction.]

Les conclusions du paragraphe précédent sont inchangées en ce qui concerne la dispersion des lignes neutres. Par contre, le déphasage $\Phi$ n'est plus rigoureusement proportionnel à $E$ pour la longueur d'onde $\lambda_{0}$. De la mesure de $\Phi$ en fonction de $E$, il serait possible de déduire l'ordre de grandeur des $c_{i j}$.

En fait, jusqu'à présent, nous avons toujours trouvé une relation linéaire entre $\Phi$ et $E$, dans la limite de la précision de nos mesures. De même, l'inversion du signe de E n'apporte pas de changement significatif, et nous n'avons pas observé de dissymétrie comparable à celles que rapportent Myers et Powell pour $\mathrm{ZnS}$ et $\mathrm{ZnTe}$ [12]. Il faut d'ailleurs remarquer que les mesures d'effet électro-optique faites sans tenir compte de la biréfringence accidentelle superposent 2 causes d'erreur : la rotation des lignes neutres et la non-linéarité de $\Phi$ en fonction de $E$. Les cristaux de Myers et Powell présentaient sans doute une forte biréfringence naturelle, ne possédant peut être pas les mêmes caractéristiques que les nôtres. Nous avons en effet toujours utilisé des cristaux de la meilleure qualité cristalline possible [9], en prenant soin d'éliminer la biréfringence aléatoire due à des contraintes, afin de ne conserver que celle qui est due aux défauts qui apparaissent systématiquement en cours de croissance.

Des mesures précises de l'influence du champ électrique sont en cours, afin d'essayer de préciser le tenseur d'imperméabilité.

8. Conclusion. - La modulation d'un faisceau lumineux par effet électro-optique du $\mathrm{ZnSe}$ peut être fortement perturbée par la biréfringence due aux défauts de structure. L'orientation du cristal et la longueur d'onde doivent être choisies de manière à minimiser la dispersion des lignes neutres avec le champ. Dans le cas des cristaux que nous avons étudiés, les meilleurs résultats sont obtenus avec un champ et un faisceau lumineux perpendiculaires à l'axe [110], et une longueur d'onde de $4930 \AA$.

\section{Bibliographie}

[1] Buhrer, C. F., Bloom, L. R., Baird, D. H., Appl. Opt. 2 (1963) 839.

[2] Kaminow, I. P.,,Turner, E. H., Proc. IEEE 54 (1966) 1374.

[3] Zheludev, I. S., Sov. Phys. 9 (1966) 97.

[4] Namba, S., J. Opt. Soc. Am. 51 (1961) 76.

[5] Baillou, J., Lemercier, M., Rivaud, G., Pavis, B., Revue Phys. Appl. 8 (1973) 121.

[6] Lee, R. W., J. Opt. Soc. Amer. 59 (1969) 1574.

[7] Ahmed, M. S., Ley, J. M., J. Phys. D : Appl. Phys. 4 (1971) 1637.
[8] Mohler, E., Thomas, B., Phys. Status Solidi (b) 79 (1977) 509.

[9] Rivaud, G., Pavis, B., Lemercier, M., Grilhe, J., J. Microsc. 19 (1974) 113

[10] Daunay, J., Baillou, J., Lemercier, M., Daunay, Jac., Bugnet, P., Batailler, G., Phys. Status Solidi (a) 37 (1976) 521.

[11] Daunay, J., Revue Phys. Appl. 11 (1976) 739.

[12] Myers, R. A., Powell, C. G., Appl. Phys. Lett. 9 (1966) 326. 